\title{
Spinal Enumeration by Morphologic Analysis of Spinal Variants: Comparison to Counting in a Cranial-To-Caudal
} Manner

\author{
Sam Yun, MD, Sekyoung Park, MD, Jung Gu Park, MD, Jin Do Huh, MD, Young Gyung Shin, MD, \\ Jong Hyouk Yun, MD \\ All authors: Department of Radiology, Kosin University Gospel Hospital, Busan 49267, Korea
}

Objective: To compare the spinal enumeration methods that establish the first lumbar vertebra in patients with spinal variants.

Materials and Methods: Of the 1446 consecutive patients who had undergone computed tomography of the spine from March 2012 to July 2016, 100 patients (62 men, 38 women; mean age, 47.9 years; age range, 19-88 years) with spinal variants were included. Two radiologists (readers 1 and 2) established the first lumbar vertebra through morphologic analysis of the thoracolumbar junction, and labeled the vertebra by counting in a cranial-to-caudal manner. Inter-observer agreement was established. Additionally, reader 1 detected the 20th vertebra under the assumption that there are 12 thoracic vertebra, and then classified it as a thoracic vertebra, lumbar vertebra, or thoracolumbar transitional vertebra (TLTV), on the basis of morphologic analysis.

Results: The first lumbar vertebra, as established by morphologic analysis, was labeled by each reader as the 21st segment in $65.0 \%$ of the patients, as the 20th segment in $31.0 \%$, and as the 19 th segment in $4.0 \%$. Inter-observer agreement between the two readers in determining the first lumbar vertebra, based on morphologic analysis, was nearly perfect ( $\kappa$ value: 1.00$)$. The 20th vertebra was morphologically classified as a TLTV in $60.0 \%$ of the patients, as the first lumbar segment in $31.0 \%$, as the second lumbar segment in $4.0 \%$, and as a thoracic segment in $5.0 \%$.

Conclusion: The establishment of the first lumbar vertebra using morphologic characteristics of the thoracolumbar junction in patients with spinal variants was consistent with the morphologic traits of vertebral segmentation.

Keywords: Spine; Anatomic variation; Lumbar vertebra; Enumeration; Thoracolumbar transitional vertebra;

Computed tomography

\section{INTRODUCTION}

The most common morphology observed in the human

Received November 15, 2017; accepted after revision May 6, 2018. Corresponding author: Sekyoung Park, MD, Department of Radiology, Kosin University Gospel Hospital, 262 Gamcheon-ro, Seo-gu, Busan 49267, Korea.

- Tel: (8251) 990-6341 - Fax: (8251) 255-2764

- E-mail: cordialsk@kosin.ac.kr

This is an Open Access article distributed under the terms of the Creative Commons Attribution Non-Commercial License (https://creativecommons.org/licenses/by-nc/4.0) which permits unrestricted non-commercial use, distribution, and reproduction in any medium, provided the original work is properly cited. spine is 24 presacral mobile vertebra, including seven cervical, 12 thoracic, and five lumbar vertebra. Although the number of cervical vertebra is fixed at seven, the number of thoracic and lumbar vertebra can vary (1-7).

Correct numbering of the vertebra is critical for avoiding operative errors. However, accurate enumeration can be challenging due to spinal variants, including anomalies in the total number or distribution of vertebra, transitional vertebra (e.g., thoracolumbar transitional vertebra [TLTV] and lumbosacral transitional vertebra [LSTV]), and other anomalies (6, 8-12). A high percentage of errors occur in patients with spinal variants $(2,13-15)$. Although correct numbering of the LSTV has been documented in previous 
literature $(6,9,11,12)$, less information is available regarding the numbering of other spinal variants $(6,16,17)$.

Therefore, the purpose of this study was to compare spinal enumeration methods used to establish the first lumbar vertebra in patients with spinal variants.

\section{MATERIALS AND METHODS}

\section{Patients}

Our Institutional Review Board approved this study, and the requirement for informed consent was waived. This study was a retrospective review of 1446 consecutive patients who underwent $\mathrm{CT}$ of the spine, including the thoracolumbar and lumbosacral junctions, for back pain between March 2012 and July 2016. We excluded 1346 patients who did not have spinal variants $(n=1269)$ or additional images ( $n$ $=77$ ) (i.e., whole-spine radiographs, magnetic resonance [MR] imaging scans or computed tomography [CT] imaging scans) that would allow us to count the number of presacral mobile vertebra (Fig. 1A). Posteroanterior chest and supine abdominal radiographs were regarded as wholespine radiographs because these images could be used to count the number of presacral mobile vertebra (Fig. 2A, B). Although it was not possible to count down from the first cervical vertebra, the number of cervical vertebra is known to be fixed at seven (1-7). Therefore, on the posteroanterior chest and supine abdominal radiographs, the number of presacral mobile vertebra could be determined by counting down from the first rib-bearing thoracic vertebra and adding the seven cervical vertebra. In addition, posteroanterior chest radiographs included the cervicothoracic junctions. Thus, the first rib could be differentiated from a cervical rib because the former arises from a superiorly directed transverse process, while the latter articulates from an inferiorly directed transverse process (18). For the same reason, chest $\mathrm{CT}$ and abdominal $\mathrm{CT}$ images were also regarded as whole-spine CT scans. The existence of spinal variants in each case was evaluated by a radiologist, based on axial spine CT with curved planar reformatting (CPR) images and images to determine the number of presacral mobile vertebra (i.e., both posteroanterior chest and supine abdominal radiographs, both chest $\mathrm{CT}$ and abdominal CT images, whole-spine radiographs, MR imaging, or CT imaging). Spinal variants included the following: 1) alteration of the typical distribution of 12 thoracic and five lumbar vertebra (Fig. 3), 2) anomalous total number of presacral mobile vertebra (e.g., 22, 23, or 25) (Fig. 1), and 3) TLTV or LSTV (Fig. 2). Park et al. (19) suggested differentiating vertebral segments at the thoracolumbar junction as TLTV or non-TLTV (thoracic or lumbar) segments on the basis of definitive criteria for differentiating most caudal ribs in consideration of several variants of the thoracolumbar junction. In accordance with the previous literature $(13,19)$, a thoracic vertebra is defined by the presence of paired ribs that are $3.8 \mathrm{~cm}$ or greater in length and originate from the facet at the pedicle (or the body) of the vertebra (Figs. 1B, C, 2C, 3C). A lumbar vertebra

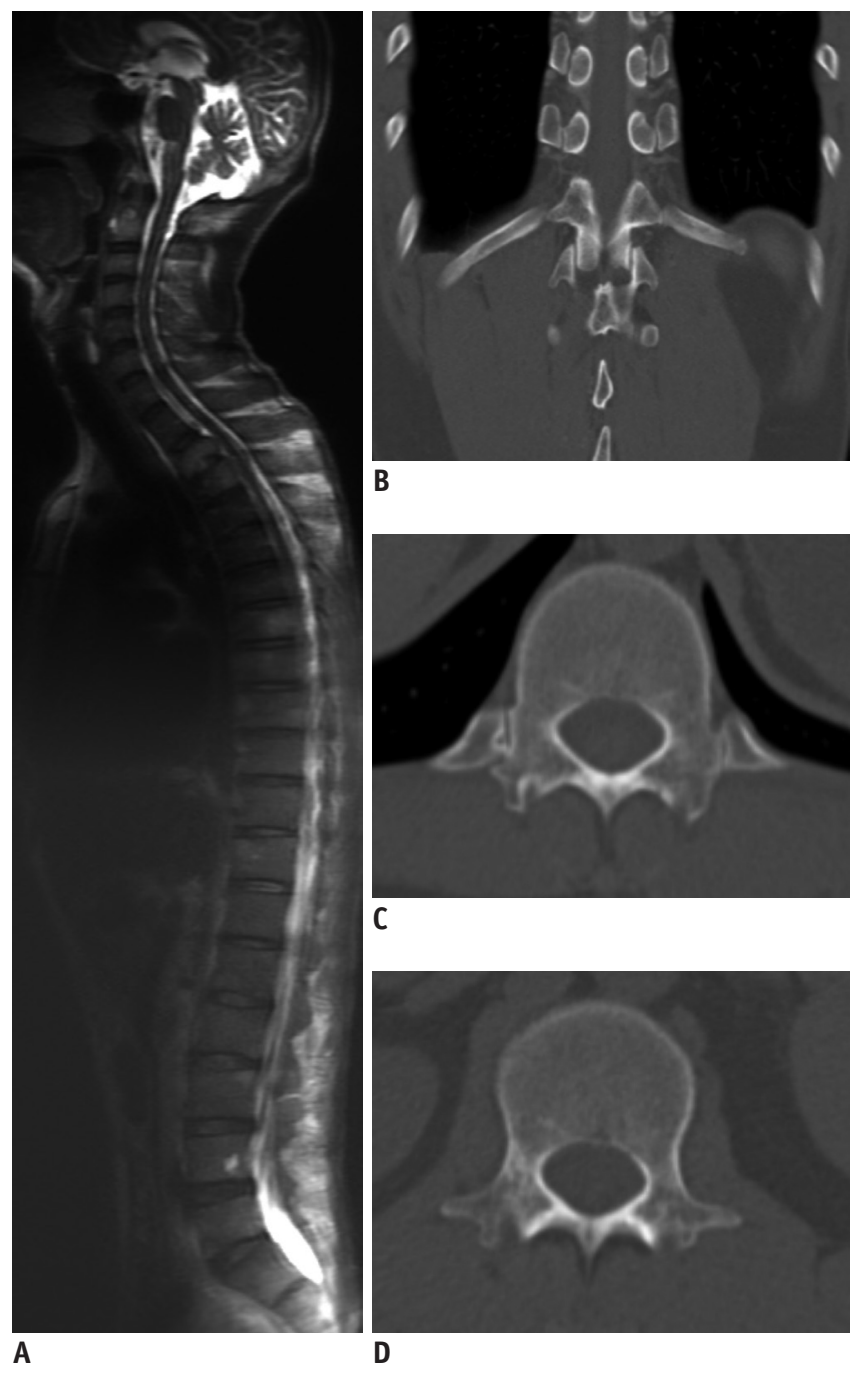

Fig. 1. 47-year-old woman with anomalous total number of vertebra.

A. Sagittal T2-weighted fast spin-echo magnetic resonance image of whole spine shows 25 presacral mobile vertebra. B, C. Coronal CT image (B) showing 20th vertebra, with paired ribs that are 3.8 $\mathrm{cm}$ or greater in length. Paired ribs originate from facet at pedicle of 20th vertebra on axial CT image (C). Therefore, 20th vertebra is morphologically thoracic vertebra. D. On axial CT image, 21st vertebra morphologically appears to be first lumbar vertebra. Thus, this patient has 25 presacral mobile vertebra with seven cervical, 13 thoracic, and five lumbar vertebra. CT = computed tomography 
was identified as a vertebra without articulating ribs, without facets for ribs on the pedicle (or the body of the vertebra), and with fused transverse processes (Figs. 1D, 2F, 3D) (13). TLTV were defined as any segments that did not meet the criteria for thoracic or lumbar vertebra in the thoracolumbar junction (Fig. 2D) (19). CPR images were
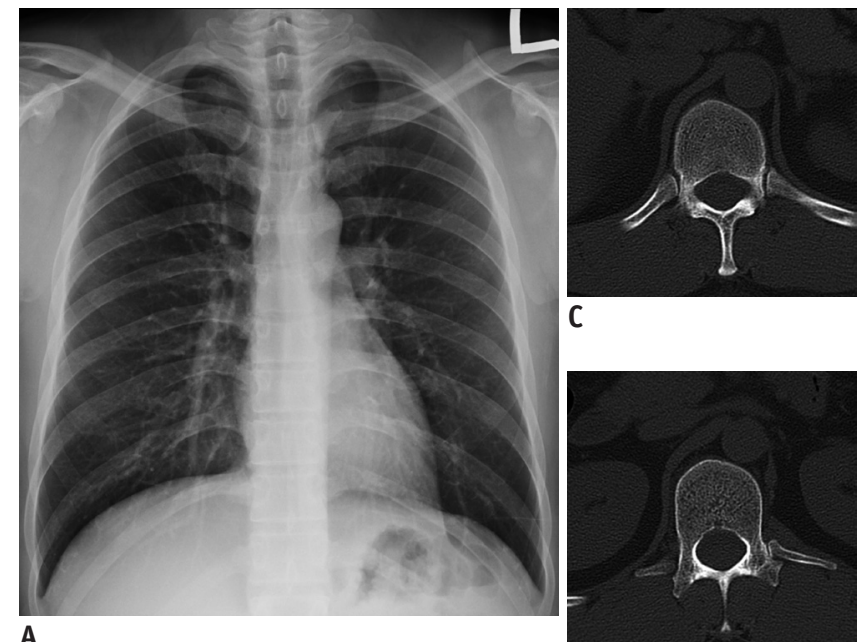

C
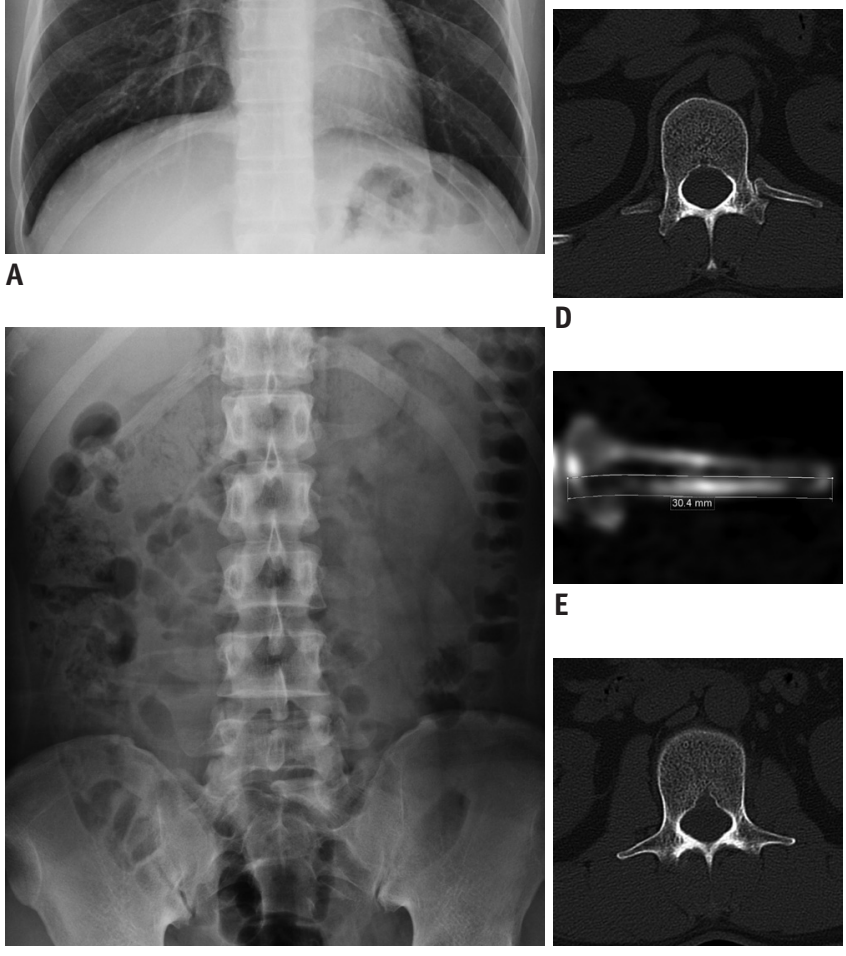

D

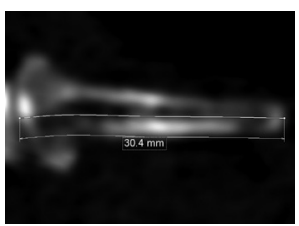

E

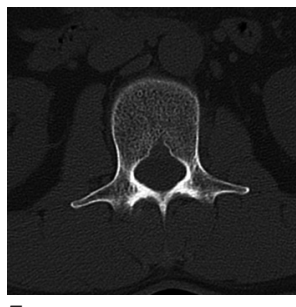

$\mathrm{F}$

Fig. 2. 38-year-old man with TLTV.

A, B. Posteroanterior chest radiograph (A) and supine abdominal radiograph (B) demonstrate 24 presacral mobile vertebra. C. Axial CT image showing 19th vertebra, with paired ribs that are $3.8 \mathrm{~cm}$ or greater in length and originate from facet at pedicle. Therefore, 19th vertebra is morphologically thoracic vertebra. D. On axial CT image, 20th vertebra, which has short rib on left side and accessory ossification center on right side, is TLTV. E. On curved planar reformatted image of left rib of 20th vertebra, rib length is measured by drawing line at midpoint of rib width from proximal head of rib to distal body. Rib measures $30.4 \mathrm{~mm}$ and is classified as short rib. F. On axial CT image, 21st vertebra, exhibiting both fused transverse processes without articulating ribs, is identified as first lumbar vertebra. Thus, this patient has 24 presacral mobile vertebra with seven cervical, 12 thoracic, one TLTV, and four lumbar vertebra. TLTV = thoracolumbar transitional vertebra used for the accurate measurement of rib length. Rib length was measured by drawing a line at the midpoint of the rib width from the proximal head of a rib to the distal body (Fig. 2E) (19). Only type I or II LSTV, as defined by the Castellvi classification (20), were included in this study, because we focused our analysis on the number of presacral mobile vertebra. Finally, 100 consecutive patients with spinal variants were included in the study.

\section{Spinal CT}

All CT images were acquired with a dual-source CT scanner (Somatom Definition Flash; Siemens Healthcare, Erlangen, Germany). Patients were scanned with a detector configuration of $128 \times 0.6 \mathrm{~mm}$ with combined modulation (CARE Dose 4D; Siemens). Standard-of-care spinal CT imaging was performed at 275 quality reference mAs. All CT imaging was performed using the following parameters: pitch, 0.7 ; gantry rotation time, 0.5 seconds; section width,
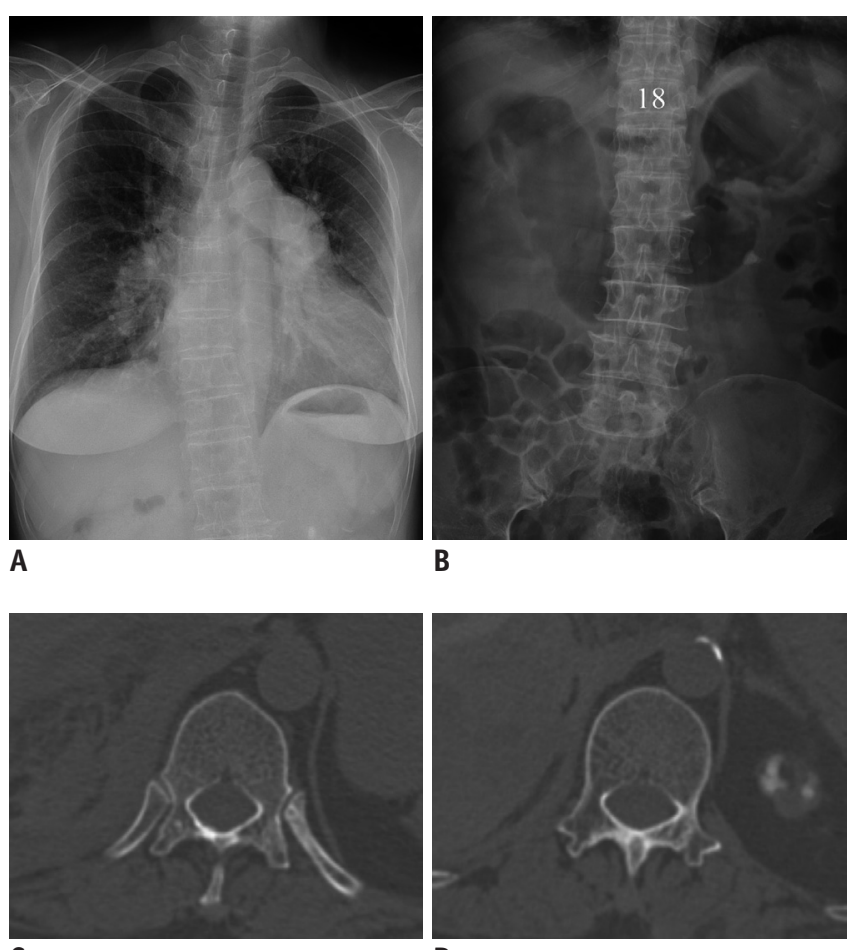

Fig. 3. 74-year-old woman with anomalous distribution of vertebra.

A, B. Posteroanterior chest radiograph (A) and supine abdominal radiograph (B) indicate presence of 24 presacral mobile vertebra. C. Axial CT image showing 18th vertebra, with paired ribs that are $3.8 \mathrm{~cm}$ or greater in length and originate from facet at pedicle. Therefore, 18th vertebra is morphologically thoracic vertebra. D. On axial CT image, 19th vertebra has both fused transverse processes and morphologically appears to be first lumbar vertebra. Thus, this patient has anomalous distribution of 24 presacral mobile vertebra with seven cervical, 11 thoracic, and six lumbar vertebra. 
$1 \mathrm{~mm}$; and reconstruction increment, $0.7 \mathrm{~mm}$.

\section{Image Analysis}

\section{Establishment of the First Lumbar Vertebra in Spinal Variants: Morphologic Analysis of the Thoracolumbar Junction}

Wigh and Anthony (21) stated that the true nature of lower vertebral segmentation can only be established with images that include the thoracolumbar junction, thereby allowing correct identification of the first lumbar vertebra. Park et al. (19) also suggested that morphologic analysis of the thoracolumbar junction may improve the accuracy of spinal enumeration. Therefore, in this study, establishment of the first lumbar vertebra through morphologic analysis was performed on images that included the thoracolumbar junction.

On the basis of the morphologic analysis of the thoracolumbar junction performed by Park et al. (19), two radiologists (readers 1 and 2) independently assessed vertebral segment types at the thoracolumbar junction, then classified them as the thoracic vertebra, lumbar vertebra, or TLTV. As described above, a lumbar vertebra was defined as a vertebra without articulating ribs, without facets for ribs on the pedicle (or the body of the vertebra), and with fused transverse processes (Figs. 1D, 2F, 3D) (13). On the basis of this definition, the first lumbar vertebra was established on axial CT images. Then, to differentiate thoracic vertebra from TLTV, the two radiologists used axial CT scans with CPR images.

During morphologic analysis of the thoracolumbar junction, each reader was blinded to the whole-spine images that allowed the number of vertebra to be counted and to the other reader's results. After the first lumbar vertebra was established, each reader labeled the first lumbar vertebra by counting in a cranial-to-caudal manner (e.g., 19th, 20th, or 21st segment), using images to determine the number of presacral mobile vertebra (i.e., both posteroanterior chest and supine abdominal radiographs, both chest CT and abdominal CT images, whole-spine radiographs, MR imaging, or CT imaging) (Figs. 1D, 2F, 3D). Inter-observer agreement between the two readers in establishing the first lumbar vertebra through morphologic analysis of the thoracolumbar junction was analyzed with kappa statistics. Kappa values were categorized as slight $(0-0.20)$, fair $(0.21-0.40)$, moderate $(0.41-0.60)$, substantial $(0.61-0.80)$, or nearly perfect (0.81-1.00) agreement (22).

\section{Comparison of Spinal Enumeration Methods for Spinal Variants}

In the traditional method of spinal numbering (4, 9-11, $23,24)$, the first lumbar vertebra is determined by counting down from the second cervical vertebra or the first thoracic vertebra, under the assumption that there are 12 thoracic vertebra. In the above method, the 20th vertebra is always the first lumbar vertebra. Accordingly, herein, the

Table 1. Characteristics of Study Patients with Spinal Variants

\begin{tabular}{|c|c|c|c|}
\hline No. of Presacral Mobile Vertebra & No. of Patients & Distribution of Vertebra & No. of Patients \\
\hline 22 & 1 & T (10)-TLTV (1)-L (4) & 1 \\
\hline \multirow{3}{*}{23} & \multirow{3}{*}{9} & $T(11)-L(5)$ & 1 \\
\hline & & T (11)-TLTV (1)-L (4) & 6 \\
\hline & & $\mathrm{T}(12)-\mathrm{L}(4)$ & 2 \\
\hline \multirow{7}{*}{24} & \multirow{7}{*}{71} & $\mathrm{~T}(11)-\mathrm{L}(6)$ & 1 \\
\hline & & T (11)-L (5)-LSTV (1) & 1 \\
\hline & & T (11)-TLTV (1)-L (4)-LSTV (1) & 5 \\
\hline & & T (11)-TLTV (1)-L (5) & 13 \\
\hline & & T (12)-TLTV (1)-L (3)-LSTV (1) & 1 \\
\hline & & T (12)-TLTV (1)-L (4) & 49 \\
\hline & & T (12)-L (4)-LSTV (1) & 1 \\
\hline \multirow{5}{*}{25} & \multirow{5}{*}{19} & $\mathrm{~T}(12)-\mathrm{L}(6)$ & 2 \\
\hline & & T (12)-L (5)-LSTV (1) & 2 \\
\hline & & T (12)-TLTV (1)-L (4)-LSTV (1) & 3 \\
\hline & & T (12)-TLTV (1)-L (5) & 7 \\
\hline & & $T(13)-L(5)$ & 5 \\
\hline
\end{tabular}

Data in parentheses are numbers of vertebra. $L=$ lumbar vertebra, $L S T V=$ lumbosacral transitional vertebra, No. $=$ number, $T=$ thoracic vertebra, TLTV $=$ thoracolumbar transitional vertebra 
Table 2. Comparison of Methods for Correct Identification of First Lumbar Vertebra

\begin{tabular}{llcl} 
& & Contents & \\
\cline { 2 - 5 } Enumeration Method & $\begin{array}{c}\text { Labeling Of First } \\
\text { Lumbar Vertebra }\end{array}$ & $\begin{array}{c}\text { Morphologic Distribution of First } \\
\text { Lumbar Vertebra, Established } \\
\text { according to Each Method }\end{array}$ & $\begin{array}{c}\text { Requires Additional } \\
\text { Whole-Spine Images }\end{array}$ \\
\hline
\end{tabular}

\section{Establishment of first lumbar vertebra,} according to morphologic analysis of thoracolumbar junction

Determination of first lumbar vertebra by counting down from second cervical vertebra or first thoracic vertebra* 21st segment, $65.0 \%$;

20th segment, $31.0 \%$; First lumbar vertebra, $100 \% \quad$ No

19th segment, $4.0 \%$

20th vertebra, $100 \%$
TLTV, 60.0\%; first lumbar vertebra, $31.0 \%$; thoracic segment, $5.0 \%$; second lumbar segment, $4.0 \%$

*Under assumption that there are 12 thoracic vertebra.

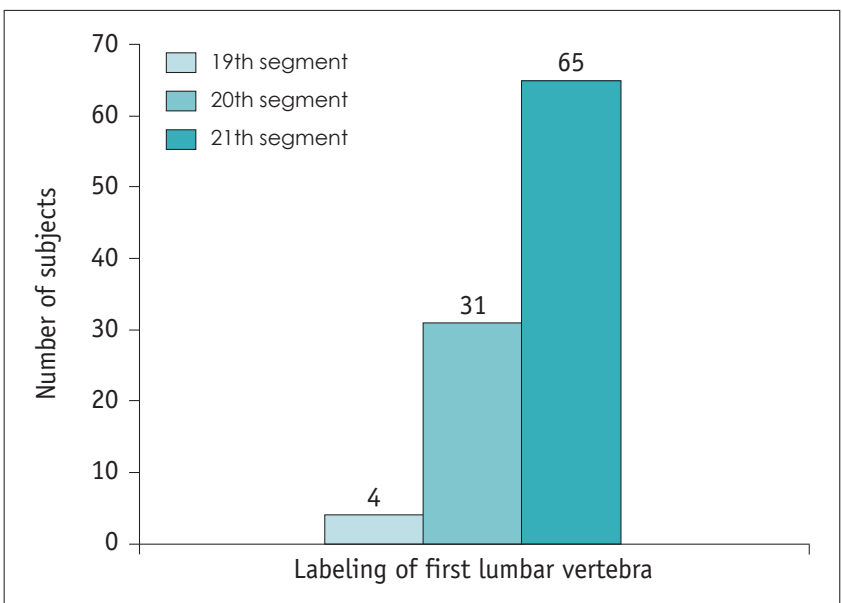

Fig. 4. Labeling of morphologic first lumbar vertebra by counting in cranial-to-caudal manner.

first lumbar vertebra identified by the above enumeration method was compared with that identified through morphologic analysis of the thoracolumbar junction. First, reader 1 detected the 20th vertebra by counting in a cranial-to-caudal manner (Figs. 1A, 2A, B). The 20th vertebra was then categorized through morphologic analysis of the thoracolumbar junction by reader 1 as thoracic, lumbar, or TLTV (Figs. 1B, C, 2D).

\section{RESULTS}

One hundred patients (62 men and 38 women) with spinal variants were included in this analysis. Mean age of the patients was 47.9 years (range, 19-88 years). Twenty-nine patients $(29.0 \%)$ had an anomalous number of presacral mobile vertebra: there were 22 presacral mobile vertebra in one patient; 23 in nine patients; and 25 in 19 patients. All patients showed anomalous distribution of vertebra. TLTV were observed in 85 patients $(85.0 \%)$ and LSTV were

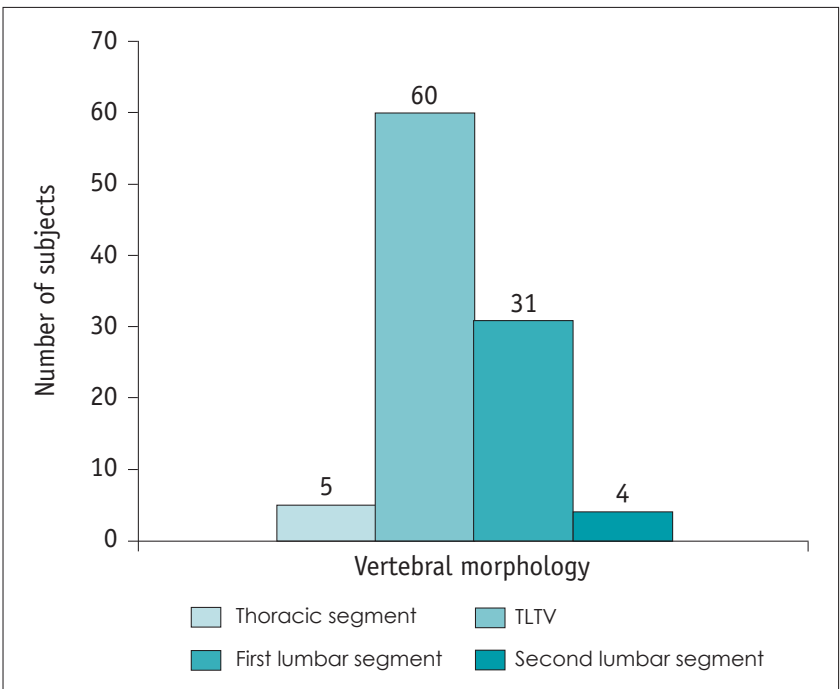

Fig. 5. Morphologic analysis of 20th vertebra.

observed in $13(13.0 \%)$. Nine patients (9\%) had both TLTV and LSTV (Table 1). A comparison of the methods used for the correct identification of the first lumbar vertebra in spinal variants is presented in Table 2 .

\section{Establishment of the First Lumbar Vertebra, according to} Morphologic Analysis of the Thoracolumbar Junction

The first lumbar vertebra, as established through morphologic analysis of the thoracolumbar junction, was labeled by each reader as the 21 st segment in $65.0 \%$ of the patients, as the 20th segment in $31.0 \%$ of the patients, and as the 19 th segment in $4.0 \%$ of the patients (Fig. 4). Interobserver agreement between the two readers in determining the first lumbar vertebra on the basis of morphologic analysis was nearly perfect ( $\kappa$ value: 1.00 ). Although there was a discrepancy in the distribution of vertebra in one case, it did not affect establishment of the first lumbar vertebra. In that case, 11 thoracic vertebra, one TLTV, and 
five lumbar vertebra were identified by reader 1 , while 12 thoracic vertebra and five lumbar vertebra were identified by reader 2 . This disagreement in values was because of a discrepancy in the measurement of the rib length by the two readers. Reader 1 considered the 19th vertebra as a TLTV, because the vertebra had paired separated bones, one of which was less than $3.8 \mathrm{~cm}$ in length, although it articulated with the facet at the pedicle. In contrast, reader 2 considered the 19th vertebra as a thoracic vertebra, because reader 2 regarded the paired ribs as being $3.8 \mathrm{~cm}$ or greater in length.

\section{Determination of the First Lumbar Vertebra by Counting Down from the Second Cervical Vertebra or the First Thoracic Vertebra}

The 20th vertebra, which was identified by counting in a cranial-to-caudal manner by reader 1 , was morphologically classified as a TLTV in $60.0 \%$ of the patients, as the first lumbar segment in $31.0 \%$ of the patients, as the second lumbar segment in $4.0 \%$ of the patients, and as a thoracic segment in $5.0 \%$ of the patients (Fig. 5).

\section{DISCUSSION}

We compared the spinal enumeration methods used to establish the first lumbar vertebra for patients with spinal variants. On the basis of morphologic analysis of the thoracolumbar junction, establishment of the first lumbar vertebra was consistent with the literal meaning of "the first lumbar vertebra."

In most previous studies, the establishment of lumbar segments in patients with LSTV has been performed with the assumption that there are seven cervical and 12 thoracic segments $(9-11,16,17,23)$. However, Carrino et al. (6) suggested that LSTV are associated with anomalies in the total number of presacral segments. Therefore, it may be inappropriate to assume that there are seven cervical and 12 thoracic segments when numbering the lumbar vertebra in patients with certain spinal variants, including LSTV. Notably, in this study, the 20th vertebra exhibited the morphologic traits of the first lumbar vertebra in just $31.0 \%$ of the patients with spinal variants. Thus far, no reports have numerically presented the aforementioned results.

As mentioned above, Wigh and Anthony (21) stated that the true nature of lower vertebral segmentation can only be established with images that include the thoracolumbar junction, thereby allowing correct identification of the first lumbar vertebra. According to Hughes and Saifuddin (12), LSTV levels can be confirmed after the first lumbar vertebra has been correctly established. Although several studies ( 9 , 11,12 ) have evaluated vertebral numbering in LSTV, studies using images that include the thoracolumbar junction have not been conducted previously. Although Carrino et al. (6) labeled the first lumbar vertebra as the first non-rib-bearing vertebra on lumbar MR imaging, they did not consider thoracolumbar junction variants. Accordingly, in this study, the first lumbar vertebra was established through morphologic analysis of the thoracolumbar junction through consideration of several variants of the thoracolumbar junction, with the method used by Park et al. (19). Thus, identification of the first lumbar vertebra by morphologic analysis of the thoracolumbar junction should be used as the reference standard to determine vertebral segments. The next step is to apply this method to the numbering of LSTV and to the evaluation of anatomic markers (e.g., aortic bifurcation, right renal artery, conus medullaris, iliolumbar ligament, and iliac crest), which are used as landmarks for the localization of lumbar vertebral segments.

In this study, inter-observer agreement in determining the first lumbar vertebra, based on morphologic analysis of the thoracolumbar junction was nearly perfect ( $\kappa$ value: 1.00). Given the definition of a lumbar vertebra (identified as a vertebra without articulating ribs, without facets for ribs on the pedicle [or the body of the vertebra], and with fused transverse processes) (13), the establishment of the first lumbar vertebra showed high reliability. Identification of the first lumbar vertebra on the basis of morphologic analysis can be performed on any axial images that include the thoracolumbar junction, without the need for images that cover the complete spinal axis.

In this study, the total number of presacral mobile vertebra varied from 22 to 25 . It is known that 23 and 25 presacral mobile vertebra are commonly observed in cases of spinal variants, while cases of the number of presacral mobile vertebra varying between 22 to 26 have been reported (25).

Lumbosacral transitional vertebra were observed in a relatively small percentage of the patients $(13.0 \%)$ in our study, while TLTV were more common (89.1\%). The only types of LSTV included in this study were type I or II LSTV, based on the Castellvi classification (20), because we focused on analyzing the number of presacral mobile vertebra. Therefore, the prevalence of LSTV was lower than that of TLTV. 
This study had several limitations. First, relatively few patients with spinal variants were evaluated. Future studies with larger sample sizes are necessary. Second, the comparison between methods in this study was not conducted in clinical practice. Therefore, future multidisciplinary studies are necessary.

In conclusion, the establishment of the first lumbar vertebra on the basis of morphologic characteristics of the thoracolumbar junction in patients with spinal variants, which was consistent with the morphologic traits of vertebral segmentation, was achieved with nearly perfect inter-observer agreement.

\section{REFERENCES}

1. Narita Y, Kuratani S. Evolution of the vertebral formulae in mammals: a perspective on developmental constraints. J Exp Zool B Mol Dev Evol 2005;304:91-106

2. Hanson EH, Mishra RK, Chang DS, Perkins TG, Bonifield DR, Tandy RD, et al. Sagittal whole-spine magnetic resonance imaging in 750 consecutive outpatients: accurate determination of the number of lumbar vertebral bodies. $J$ Neurosurg Spine 2010;12:47-55

3. Galis F. Why do almost all mammals have seven cervical vertebrae? Developmental constraints, hox genes, and cancer. J Exp Zool 1999;285:19-26

4. Akbar JJ, Weiss KL, Saafir MA, Weiss JL. Rapid MRI detection of vertebral numeric variation. AJR Am J Roentgenol 2010;195:465-466

5. Thawait GK, Chhabra A, Carrino JA. Spine segmentation and enumeration and normal variants. Radiol Clin North Am 2012;50:587-598

6. Carrino JA, Campbell PD Jr, Lin DC, Morrison WB, Schweitzer $M E$, Flanders $A E$, et al. Effect of spinal segment variants on numbering vertebral levels at lumbar MR imaging. Radiology 2011;259:196-202

7. Kier EL. Some developmental and evolutionary aspects of the lumbosacral spine. In: Gouaze A, Salamon G, eds. Brain anatomy and magnetic resonance imaging. Berlin, Heidelberg: Springer Berlin Heidelberg, 1988:116-139

8. Lee CH, Park CM, Kim KA, Hong SJ, Seol HY, Kim BH, et al. Identification and prediction of transitional vertebrae on imaging studies: anatomical significance of paraspinal structures. Clin Anat 2007;20:905-914

9. Farshad-Amacker NA, Aichmair A, Herzog RJ, Farshad M. Merits of different anatomical landmarks for correct numbering of the lumbar vertebrae in lumbosacral transitional anomalies. Eur Spine J 2015;24:600-608
10. Hahn PY, Strobel JJ, Hahn FJ. Verification of lumbosacral segments on MR images: identification of transitional vertebrae. Radiology 1992;182:580-581

11. Farshad-Amacker NA, Lurie B, Herzog RJ, Farshad M. Is the iliolumbar ligament a reliable identifier of the $L 5$ vertebra in lumbosacral transitional anomalies? Eur Radiol 2014;24:26232630

12. Hughes RJ, Saifuddin A. Numbering of lumbosacral transitional vertebrae on MRI: role of the iliolumbar ligaments. AJR Am J Roentgenol 2006;187:W59-W65

13. Wigh RE. The thoracolumbar and lumbosacral transitional junctions. Spine (Phila Pa 1976) 1980;5:215-222

14. Wigh RE. Phylogeny and the herniated disc. South Med J 1979;72:1138-1143

15. Malanga GA, Cooke PM. Segmental anomaly leading to wrong level disc surgery in cauda equina syndrome. Pain Physician 2004;7:107-110

16. Paik NC, Lim CS, Jang HS. Numeric and morphological verification of lumbosacral segments in 8280 consecutive patients. Spine (Phila Pa 1976) 2013;38:E573-E578

17. Peckham ME, Hutchins TA, Stilwill SE, Mills MK, Morrissey $B J$, Joiner EAR, et al. Localizing the $L 5$ vertebra using nerve morphology on MRI: an accurate and reliable technique. AJNR Am J Neuroradiol 2017;38:2008-2014

18. Maus TP. Spine imaging, an issue of radiologic clinics of North America, Volume 50-4, 1st ed. Philadelphia, PA: Elsevier Health Sciences, 2012:587-598

19. Park SK, Park JG, Kim BS, Huh JD, Kang H. Thoracolumbar junction: morphologic characteristics, various variants and significance. Br J Radiol 2016:20150784

20. Castellvi AE, Goldstein LA, Chan DP. Lumbosacral transitional vertebrae and their relationship with lumbar extradural defects. Spine (Phila Pa 1976) 1984;9:493-495

21. Wigh RE, Anthony HF Jr. Transitional lumbosacral discs. Probability of herniation. Spine (Phila Pa 1976) 1981;6:168171

22. Landis JR, Koch GG. The measurement of observer agreement for categorical data. Biometrics 1977;33:159-174

23. Peh WC, Siu TH, Chan JH. Determining the lumbar vertebral segments on magnetic resonance imaging. Spine (Phila Pa 1976) 1999;24:1852-1855

24. Tureli D, Ekinci G, Baltacioglu F. Is any landmark reliable in vertebral enumeration? A study of 3.0-Tesla lumbar MRI comparing skeletal, neural, and vascular markers. Clin Imaging 2014;38:792-796

25. Ten Broek CM, Bakker AJ, Varela-Lasheras I, Bugiani M, Van Dongen S, Galis F. Evo-devo of the human vertebral column: on homeotic transformations, pathologies and prenatal selection. Evol Biol 2012;39:456-471 